Mon. Not. R. Astron. Soc. 000,1012(2002) Printed 19 September $2018 \quad$ (MN LATEX style file v2.2)

\title{
Gaseous Tidal Debris found in the NGC 3783 Group $^{\star}$
}

\author{
Virginia A. Kilborn ${ }^{1,2}$, Duncan A. Forbes ${ }^{1}$, Bärbel S. Koribalski ${ }^{2}$, Sarah Brough ${ }^{1}$, \\ Katie Kern ${ }^{1,2}$ \\ ${ }^{1}$ Centre for Astrophysics \&S Supercomputing, Swinburne University, Hawthorn, VIC 3122, Australia \\ ${ }^{2}$ Australia Telescope National Facility, CSIRO, P.O. Box 76, Epping, NSW 1710, Australia
}

Accepted; Received

\begin{abstract}
We have conducted wide-field $\mathrm{H}$ I mapping of a $\sim 5.5^{\circ} \times 5.5^{\circ}$ region surrounding the NGC 3783 galaxy group, to an H I mass limit of $\sim 4 \times 10^{8} M_{\odot}$. The observations were made using the multibeam system on the Parkes 64-m radiotelescope, as part of the Galaxy Evolution Multiwavelength Study (GEMS). We find twelve H I detections in our Parkes data, four more than catalogued in HIPASS. We find two new group members, and discover an isolated region of $\mathrm{H}$ I gas with an $\mathrm{H}$ I mass of $\sim 4 \times 10^{8} M_{\odot}$, without a visible corresponding optical counterpart. We discuss the likelihood of this $\mathrm{H}$ I region being a low surface brightness galaxy, primordial gas, or a remnant of tidal debris. For the NGC 3783 group we derive a mean recession velocity of $2903 \pm 26$ $\mathrm{km} \mathrm{s}^{-1}$, and a velocity dispersion of $190 \pm 24 \mathrm{~km} \mathrm{~s}^{-1}$. The galaxy NGC 3783 is the nearest galaxy to the luminosity weighted centre of the group, and is at the group mean velocity. From the X-ray and dynamical state of this galaxy group, this group appears to be in the early stages of its evolution.
\end{abstract}

Key words: galaxies: cluster: general - galaxies: evolution - galaxies: irregular

\section{INTRODUCTION}

The physical processes operating in galaxy groups are likely to be somewhat different to those operating in galaxy clusters. For example, the intragroup medium is significantly less dense than the intracluster medium and galaxy motions slower - suggesting that ram pressure stripping is less effective in groups than in clusters (Mulchaey \& Zabludoff 1998). On the other hand, as relative galaxy motions are smaller, gravitational interactions will be more effective and outright mergers more common in groups (Zabludoff \& Mulchaey 1998).

Neutral hydrogen (H I) observations are a particularly useful method of probing the relative importance of ram pressure stripping and tidal interactions. The $\mathrm{H}$ i is typically more extended than the optical disk of a galaxy, and thus is more sensitive to tidal interactions than the stars (e.g. Haynes, Giovanelli \& Chincarini 1984), and ram pressure stripping affects the gas rather than the stars in a galaxy. In addition, H I surveys can detect new, optically faint but gasrich galaxies, previously unidentified in optical surveys (e.g.

\footnotetext{
* The observations were obtained with the Australia Telescope which is funded by the Commonwealth of Australia for operations as a National Facility managed by CSIRO.
}

Kilborn et al. 2005; Banks et al. 1999). However, systematic, optically unbiased H I surveys of loose galaxy groups are few. These Hi surveys of groups have found galaxies with extended HI envelopes (Haynes 1981), some galaxies with significant H I deficiencies (Kilborn et al. 2005; Omar \& Dwarakanath 2005) and that there are no intergalactic H I clouds in groups similar to the Local Group (Pisano et al. 2004; Zwaan 2001).

Tidal interactions between gas-rich galaxies (e.g. Barnes \& Hernquist 1986; Toomre \& Toomre 1972) are known to produce H i bridges (e.g. Koribalski \& Manthey 2005; Koribalski \& Dickey 2004; Yun, Lo \& Ho 1994; Li \& Seaquist 1994), tails (e.g. Hibbard et al. 2001b; Hibbard \& Yun 1999) or even 'clouds' that are spatially distinct from the host galaxy (e.g. Bekki, Koribalski \& Kilborn 2005a; Bekki et al. 2005b; Ryder et al. 2001; Schneider 1985). Such H I clouds, with no obvious optical counterpart, have been detected in clusters (Oosterloo \& van Gorkom 2005; Davies et al. 2004; Giovanelli \& Haynes 1989) but their origin is not always immediately obvious (Bekki et al. 2005a; Minchin et al. 2005; Djorgovski 1990). Determining the origin of H I 'clouds' is vital. For example, cosmological n-body simulations of the Local Group predict many more dark matter halos, than galaxies are optically observed in the Local Group (Moore et al. 1999; Klypin et al. 1999). Could these halos be de- 


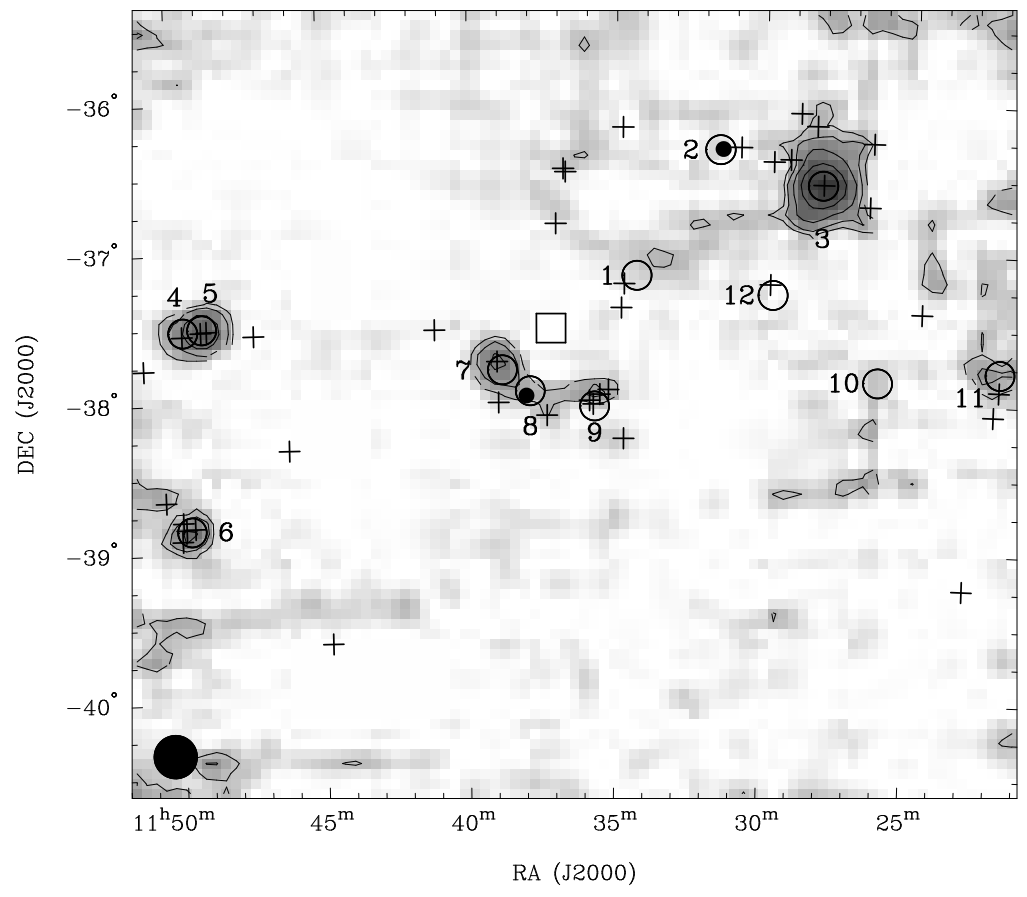

Figure 1. Velocity integrated total H I map of the NGC 3783 group. The contour levels are 2, 4, 8, 16, 32, 64 Jy km s ${ }^{-1}$. The large open circles mark the HI detections (numbered 1-12), the crosses mark galaxies with known redshifts in the region from NED and 6dFGS DR2 with velocities between $2500-3500 \mathrm{~km} \mathrm{~s}^{-1}$. The filled circles mark the positions of the previously uncatalogued H detections GEMS_N3783_2 and GEMS_N3783_8 from the ATCA. The open square marks the luminosity-weighted centre of the group. Galaxy \#7 is NGC 3783 itself, which lies at the centre of an extended X-ray halo. The Parkes beam is shown in the bottom left corner.

tected in an H i survey? Despite deep H i surveys of groups (Pisano et al. 2004; Zwaan 2001), and the HIPASS southern sky H I survey (Meyer et al. 2004), we are yet to identify any H I sources that are primordial, i.e. are not associated with a star forming galaxy.

This paper describes H I observations of the NGC 3783 group, and in particular, the discovery of several new group members, and a region of $\mathrm{HI}$ emission that has no optical counterpart. This work is part of a larger investigation into the properties of galaxies in groups, the Group Evolution Multiwavelength Study (GEMS). GEMS is a large survey of about 60 groups that were selected to have previous ROSAT PSPC X-ray observations (see Forbes et al. 2006). Osmond \& Ponman (2004; hereafter OP04) detail the selection criteria and group properties for the 60 groups. We have made wide-field H I observations of 16 GEMS groups with the Parkes telescope. These observations will allow us to characterise the $\mathrm{H}$ I content of loose groups, and the relationship between $\mathrm{H}$ I content and X-ray emission. Kilborn et al. $(2005,2006)$ describe the H I sub-sample and characteristics.

The NGC 3783 group was first identified by Giuricin et al. (2000), who found three galaxies including the wellstudied Seyfert galaxy NGC 3783. The group lies at a distance of $36 \mathrm{Mpc}$ (OP04); at this distance, $1^{\prime \prime}=174.5 \mathrm{pc}$. OP04 find extended X-ray emission surrounding NGC 3783, out to a radius of $69 \mathrm{kpc}$ (see Section 3.2 for details).

We briefly discuss our $\mathrm{H}$ I observations and data reduction in Section 2, and the results from the wide-field $\mathrm{HI}$
Table 1. Parkes narrow-band datacube parameters for the NGC 3783 group.

\begin{tabular}{lc}
\hline Centre $\left[\alpha(\mathrm{h} \mathrm{m}), \delta\left({ }^{\circ}\right)(\mathrm{J} 2000)\right]$ & $11: 37,-37: 53$ \\
Gridded beam size $\left[{ }^{\prime}\right]$ & 15.5 \\
Cube size $\left[{ }^{\circ}\right]$ & $5.5 \times 5.5$ \\
Velocity range $\left[\mathrm{km} \mathrm{s}^{-1}\right]$ & $1979-3615$ \\
Channel width $\left[\mathrm{km} \mathrm{s}^{-1}\right]$ & 1.65 \\
Velocity resolution $\left[\mathrm{km} \mathrm{s}^{-1}\right]$ & 2.6 \\
rms noise per channel $\left[\mathrm{mJy} \mathrm{beam}^{-1}\right]$ & 26 \\
\hline
\end{tabular}

imaging in Section 3. High resolution observations of NGC 3783 itself, and a nearby dwarf galaxy are presented in Section 4 , and we discuss the discovery of a region of intra-group H I gas in Section 5. We finish with discussions in Section 6, and conclusions in Section 7. Throughout we use a heliocentric velocity in the optical convention.

\section{OBSERVATIONS AND DATA REDUCTION}

\subsection{Parkes HI Observations}

Wide-field H I observations for the NGC 3783 group were conducted as part of the GEMS H I study (Kilborn et al. 2005, 2006). The observations were made using the inner seven beams of the $20-\mathrm{cm}$ multibeam system on the Parkes 64-m telescope in NSW, Australia. The area observed covers $\sim 5.5^{\circ} \times 5.5^{\circ}$ centred on the NGC 3783 galaxy group. 
Table 2. Details of high resolution ATCA H i data obtained for galaxies in the NGC 3783 group. The columns are as follows: (1) GEMS galaxy number; (2) New observation (O), or archive data used (A); (3) Observation dates; (4) ATCA array used for the observations; (5) Total integration time obtained for source in hours; (6) ATCA beam size in $\operatorname{arcsec}^{2}$; (7) Spectral resolution of the final datacube $\left(\mathrm{km} \mathrm{s}^{-1}\right)$; (8) rms noise level per channel of the final datacube $\left(\mathrm{mJy}\right.$ beam $\left.{ }^{-1}\right)$.

\begin{tabular}{|c|c|c|c|c|c|c|c|}
\hline $\begin{array}{l}\text { No. } \\
\text { (1) }\end{array}$ & $\begin{array}{c}\text { O or } \mathrm{A} \\
(2)\end{array}$ & $\begin{array}{l}\text { Observation Dates } \\
\text { (3) }\end{array}$ & $\begin{array}{l}\text { Array/s } \\
\text { (4) }\end{array}$ & $\begin{array}{l}\text { Int. Time } \\
(\mathrm{h}) \\
(5)\end{array}$ & $\begin{array}{c}\text { Beam size } \\
\left({ }^{\prime \prime} \times{ }^{\prime \prime}\right) \\
(6)\end{array}$ & $\begin{array}{r}\text { Spectral Res. } \\
\left(\mathrm{km} \mathrm{s}^{-1}\right) \\
(7)\end{array}$ & $\begin{array}{r}\mathrm{rms} \\
(\mathrm{mJy} \text { beam }-1) \\
(8)\end{array}$ \\
\hline 1 & $\mathrm{O}$ & 2006 Jan 28 & $750 \mathrm{D}$ & 1.6 & $102 \times 34$ & 6.6 & 5.5 \\
\hline 2 & $\mathrm{O}$ & 2004 March 03, 2006 Jan 25-26 & $750 \mathrm{~A}, 750 \mathrm{D}$ & 20 & $88 \times 50$ & 13.2 & 2.8 \\
\hline 3 & $\mathrm{O}$ & 2006 Jan 27 & $750 \mathrm{D}$ & 1.35 & $83 \times 47$ & 6.6 & 4.0 \\
\hline 4,5 & $\mathrm{~A}$ & 2001 July 09 & 375 & 1 & $58 \times 145$ & 6.6 & 6.0 \\
\hline 6 & $\mathrm{O}$ & 2004 Nov 12 & $750 \mathrm{C}$ & 4.5 & $98 \times 55$ & 3.3 & 5.0 \\
\hline 7 & $\mathrm{~A}$ & $\begin{array}{l}1993 \text { Oct } 09-10,1993 \text { Sept } 29-30 \text {, } \\
1996 \text { Oct } 17-18\end{array}$ & $1.5 \mathrm{D}, 750 \mathrm{D}, 1.5 \mathrm{~A}$ & 18.1 & $50 \times 50$ & 10.0 & 1.5 \\
\hline
\end{tabular}

The velocity range of the data is from 1979 to $3615 \mathrm{~km} \mathrm{~s}^{-1}$. The observational strategy and subsequent data reduction are described in detail in Kilborn et al. (2005, 2006). Approximately 25 hours of data were obtained for this region. We used the aips ++ routines LIVEDATA and GRIDZILLA to reduce the data, in the same way that is explained in detail in Kilborn et al. (2005).

For this particular dataset, approximately $30 \%$ of the data was affected by solar interference, which produces a 'ripple' effect in the reduced datacube. While the $\mathrm{H}$ I detections in the cube are visible, the cube contains a number of artifacts and the data quality is variable both spatially and in velocity. Thus, a subsequent datacube was made from the remaining $70 \%$ of ripple-free scans only. Although the rms noise for this datacube is slightly higher, the artifacts that had appeared in the original datacube are absent, and the rms is constant throughout the new datacube. The new, ripple-free, $\mathrm{H}$ I datacube has an rms noise of $26 \mathrm{mJy}^{\text {beam }}{ }^{-1}$ per channel, and the parameters for the datacube are given in Table 1 We smoothed this datacube to a velocity resolution of $13.2 \mathrm{~km} \mathrm{~s}^{-1}$, and the rms noise in the smoothed cube is $11.8 \mathrm{mJy}$ beam $^{-1}$ per channel. For the distance of this group, this gives a $3 \sigma \mathrm{H}$ I mass limit of $3.8 \times 10^{8} M_{\odot}$ (assuming a Gaussian profile, and an $\mathrm{H}$ I velocity width of 50 $\mathrm{kms}^{-1}$ ). The smoothed $\mathrm{H}_{\mathrm{I}}$ datacube was searched for $\mathrm{H} \mathrm{I}$ detections visually, using the KARMA visualisation package (see Kilborn et al. 2005, 2006, for cube searching and source parameterisation details).

\subsection{ATCA Observations}

We obtained higher spatial resolution observations from the Australia Telescope Compact Array (ATCA) both through dedicated observing programs, and from the ATCA archive. Standard MIRIAD routines were applied to reduce the data, and 'natural' weighting was used in each case. We used the primary flux calibrator $1934-638$ for all the observations. The full-width half-power primary beam of the ATCA is $\sim 33^{\prime}$ at $1.4 \mathrm{GHz}$. The observational details are summarised in Table 2

\section{HI CONTENT AND CHARACTERISTICS OF THE NGC 3783 GROUP}

\subsection{H I Characteristics of the group}

Figure 1shows a velocity integrated H I map from the Parkes data for the NGC 3783 group. The H I observations reveal a very loose grouping, that is not symmetrically distributed around the galaxy NGC 3783. We detect twelve sources in our Parkes H I datacube, including the galaxy NGC 3783 itself. Table 3 lists the $\mathrm{H}$ I position, systemic velocity and H I mass (assuming a distance of $36 \mathrm{Mpc}$ ) for each of the NGC 3783 group members as measured in the Parkes HI datacube, as well as their optical counterparts and 6dFGS DR2 velocity (see below). Their H I spectra are shown in Figure 2 and additional Hi properties will be presented in Kilborn et al. (2006).

We used the NASA Extragalactic Database (NED) and the 6dF Galaxy Survey Data Release 2 (6dFGS DR2; Jones et al. 2005) to find previously catalogued galaxies in the region of the $\mathrm{H}$ I datacube. Those galaxies in the region with a known redshift between $2500-3500 \mathrm{~km} \mathrm{~s}^{-1}$ are indicated with a cross on Figure 11 We searched a region of 10 arcminutes around the position of each $\mathrm{H}$ I detection to find the corresponding optical counterparts. These are listed in Table 3

Two of the Hi detections (GEMS_N3783_2, and GEMS_N3783_8) were not previously catalogued in any optical catalogues (see Section 4 and Section 5 for details). One H I detection, GEMS_N3783_10, provides the first velocity for ESO 319- G 020, placing it at the group velocity. Details of the new group members are summarised in Table 5

There were four Hi detections that have several optically catalogued sources (with velocities near that of the H I source) within our 10 arcminute search region. Those HI detections are GEMS_N3783_6, GEMS_N3783_4, GEMS_N3783_5 and GEMS_N3783_9. As described in Section 2.2 we observed GEMS_N3783_6 in higher spatial resolution at the ATCA. We found the H I was associated with at least two of the galaxies close to the central position of the Parkes H I detection (ESO 320-G 023 and ESO 320-G 026), and a tentative detection was made of the other galaxy in the region, 6dF J1149529-385431. Archive ATCA data for GEMS_N3783_4 and GEMS_N3783_5 was available. These two $\mathrm{H}$ I detections lie very close to one another in projection 
GEMS_N3783_1

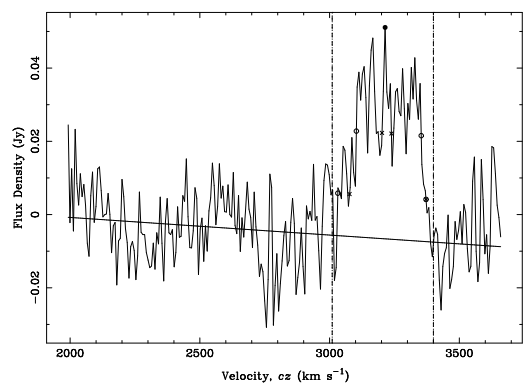

GEMS_N3783_4

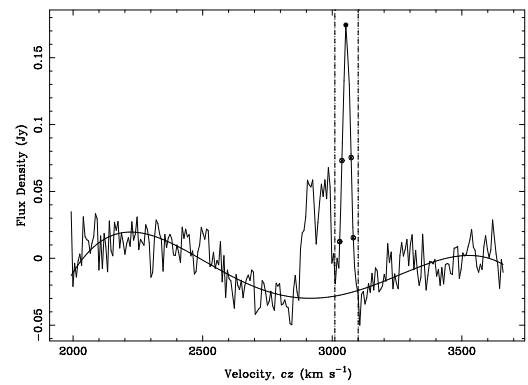

GEMS_N3783_7

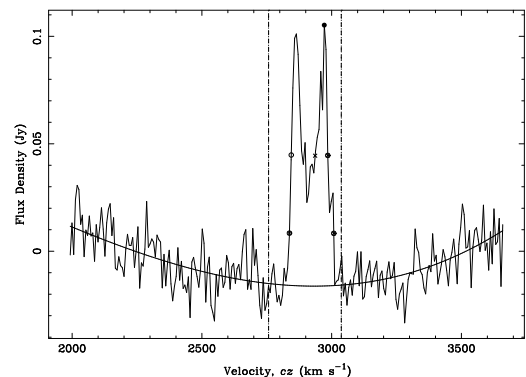

GEMS_N3783_10

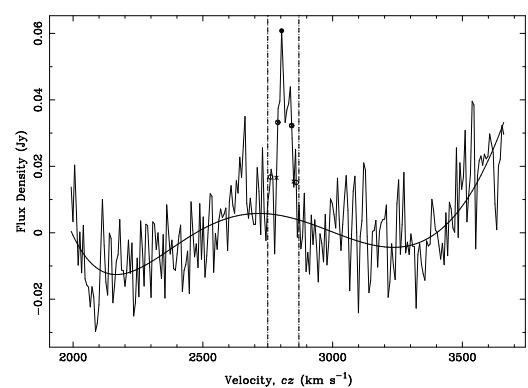

GEMS_N3783_2

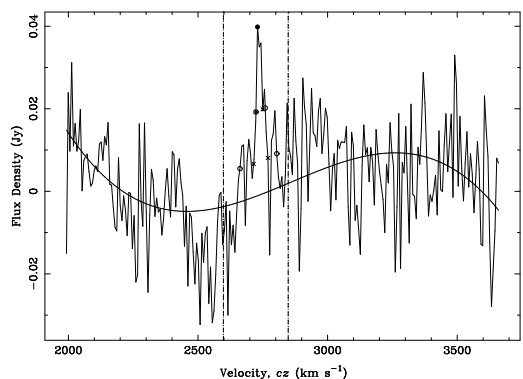

GEMS_N3783_5

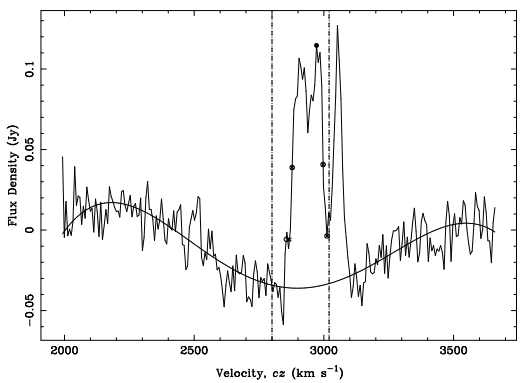

GEMS_N3783_8

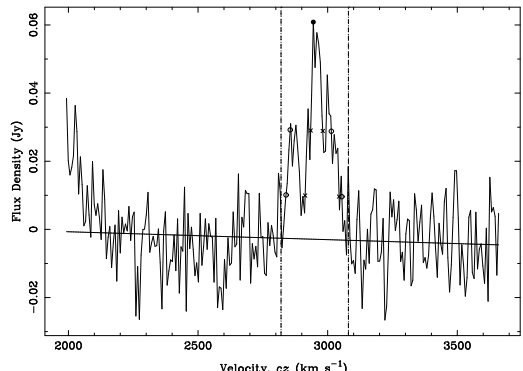

GEMS_N3783_11

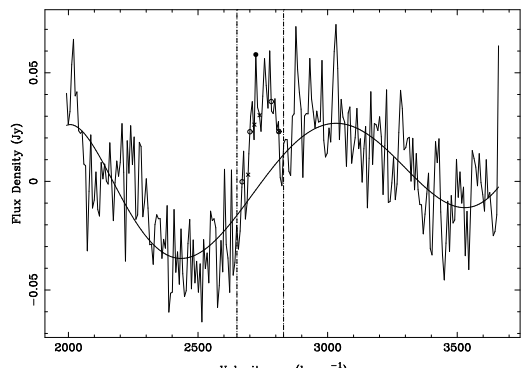

GEMS_N3783_3

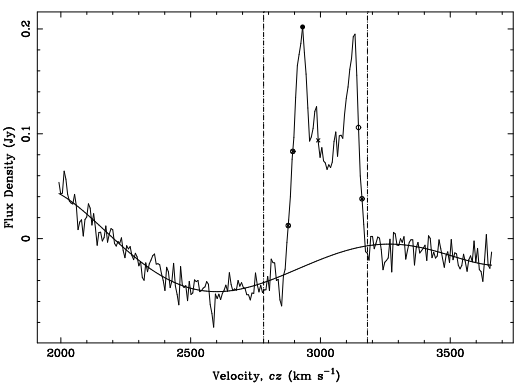

GEMS_N3783_6

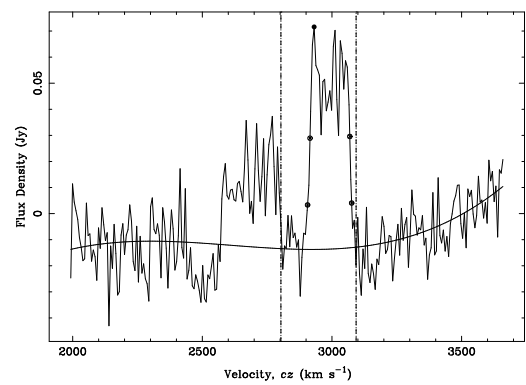

GEMS_N3783_9

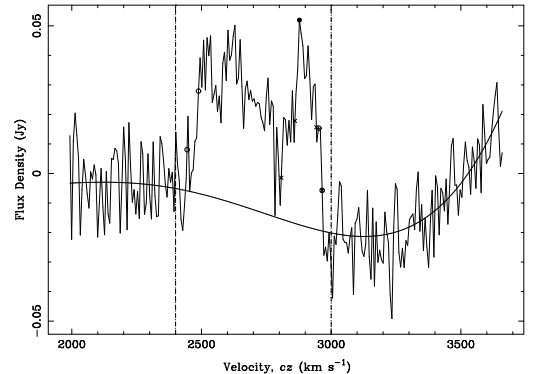

GEMS_N3783_12

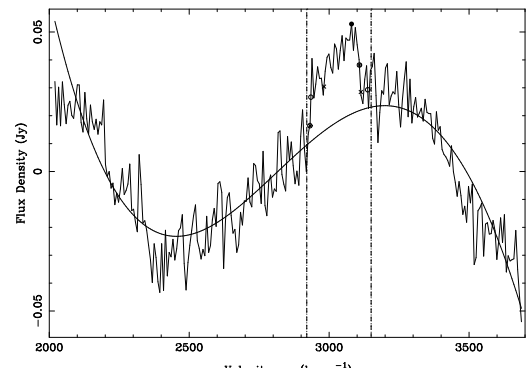

Figure 2. H I spectra of the twelve galaxies detected in our NGC 3783 survey. The spectra were Hanning smoothed to a velocity resolution of $6.6 \mathrm{~km} \mathrm{~s}^{-1}$. The fitted baseline is shown and the H I peak flux density is marked with a filled circle. The $w_{20}$ and $w_{50}$ velocity widths are shown by the open circles (outer fit), and crosses (inner fit). The velocity region between the vertical lines in the spectra was disregarded in the baseline fit.

(separation of $\sim 2^{\prime}$ ), but are separated in velocity in the Parkes data by $114 \mathrm{~km} \mathrm{~s}^{-1}$. The ATCA observations confirm that GEMS_N3783_4 is associated with AM1147-371. However, the beam size of the ATCA observations was such that it was not possible to confirm whether GEMS_N3783_5 is associated with one, or both of ESO 378-G 023 and NGC 3903, so we list both in Table 3 We do not have any high resolution imaging of GEMS_N3783_9, so we list all 4 nearby optical candidates in Table 3

The HIPASS catalogue, HICAT, contains eight of the 
Table 3. H I detections from our NGC 3783 Parkes datacube.

\begin{tabular}{|c|c|c|c|c|c|c|}
\hline $\begin{array}{l}\text { No. } \\
\text { (1) }\end{array}$ & $\begin{array}{c}\alpha, \delta(\mathrm{J} 2000) \\
{[\mathrm{h} \mathrm{m} \mathrm{s}],\left[\begin{array}{ll}0 & \prime \prime\end{array}\right]} \\
(2)\end{array}$ & $\begin{array}{r}v_{s y s} \\
{\left[\mathrm{~km} \mathrm{~s}^{-1}\right]} \\
(3)\end{array}$ & $\begin{array}{r}M_{\mathrm{HI}} \\
{\left[10^{8} M_{\odot}\right]} \\
(4)\end{array}$ & $\begin{array}{c}\text { Separation } \\
\text { [arcmin] } \\
(5)\end{array}$ & $\begin{array}{l}\text { Optical ID/s } \\
(6)\end{array}$ & $\begin{array}{l}\text { Velocity } \\
{\left[\mathrm{km} \mathrm{s}^{-1}\right.} \\
(7)\end{array}$ \\
\hline 1 & $11: 34: 18-37: 09: 39$ & $3202 \pm 7$ & $34.0 \pm 3.3$ & 6.1 & ESO 378- G 011 & 3245 \\
\hline 2 & $11: 31: 32-36: 18: 53$ & $2733 \pm 10$ & $3.8 \pm 1.3$ & $\cdots$ & $\cdots$ & $\cdots$ \\
\hline 3 & $11: 28: 06-36: 32: 45$ & $3018 \pm 2$ & $116.0 \pm 5.4$ & 0.4 & ESO 378- G 003 & 3022 \\
\hline 4 & $11: 49: 40-37: 30: 50$ & $3055 \pm 1$ & $21.0 \pm 2.3$ & 1.7 & AM1147-371 & 2964 \\
\hline \multirow[t]{2}{*}{5} & $11: 49: 01-37: 29: 47$ & $2933 \pm 2$ & $51.1 \pm 3.4$ & 1.3 & ESO 378- G 023 & 2932 \\
\hline & & & & 2.0 & NGC 3903 & 2983 \\
\hline \multirow[t]{3}{*}{6} & $11: 49: 33-38: 50: 40$ & $2991 \pm 2$ & $32.5 \pm 2.9$ & 1.8 & ESO 320- G 024 & 3037 \\
\hline & & & & 4.9 & ESO 320- G 026 & 2716 \\
\hline & & & & 5.5 & 6dF J1149529-385431 & 2708 \\
\hline 7 & $11: 38: 51-37: 47: 38$ & $2923 \pm 2$ & $38.0 \pm 2.9$ & 3.9 & NGC 3783 & 2817 \\
\hline 8 & $11: 37: 54-37: 56: 04$ & $2947 \pm 5$ & $21.0 \pm 2.5$ & $\cdots$ & $\ldots$ & $\cdots$ \\
\hline \multirow[t]{4}{*}{9} & $11: 35: 43-38: 02: 08$ & $2705 \pm 5$ & $59.5 \pm 4.1$ & 1.0 & AM 1133-374 & 2742 \\
\hline & & & & 3.0 & NGC 3749 & 2742 \\
\hline & & & & 5.2 & NGC 3742 & 2715 \\
\hline & & & & 8.6 & 2MASX J11351493-3755309 & 2823 \\
\hline 10 & $11: 26: 06-37: 51: 26$ & $2810 \pm 5$ & $7.2 \pm 1.5$ & 4.0 & ESO 319- G 020 & $\cdots$ \\
\hline 11 & $11: 21: 57-37: 46: 45$ & $2740 \pm 5$ & $12.6 \pm 2.0$ & 7.2 & ESO 319- G 015 & 2737 \\
\hline 12 & $11: 29: 43-37: 16: 59$ & $3034 \pm 7$ & $13.7 \pm 2.7$ & 4.3 & ESO 378- G 007 & 3041 \\
\hline
\end{tabular}

The columns are (1) GEMS galaxy number, (2) fitted H I centre position, (3) H I systemic velocity in the optical convention, (4) H I mass for the detection using group distance of $36 \mathrm{Mpc}$. (5) Distance of optical counterpart from centre of the HI emission. (6) Optical counterpart/s for the H I detection (7) Velocity of the optical counterpart, from 6dFGS DR2, apart from AM1147-371 where the velocity is a previous H I measurement from Matthews, Gallagher \& Littleton (1995). The errors are derived following Koribalski et al. (2004). Cols (2)-(4) are H I properties as derived from the Parkes data.

twelve galaxies we detected in our $\mathrm{H}$ I datacube (Meyer et al. 2004). Three of the detections that are not in HICAT have small Hi fluxes, and the fourth, GEMS_N3783_5, appears to have been missed in HICAT due to confusion with the nearby GEMS_N3783_4. The HIPASS rms in the region of the NGC 3783 group is $\sim 15-16$ mJy beam $^{-1}$ per channel, which higher than our GEMS data that has an rms noise of $11.8 \mathrm{mJy} \mathrm{beam}^{-1}$ per channel (smoothed to the same resolution as HIPASS).

We compare the H i fluxes between HICAT and GEMS and find there is excellent agreement for six of the eight galaxies that the two surveys have in common. There is a disagreement in $\mathrm{H}$ I flux for two of the galaxies that are in both HICAT and GEMS. The GEMS H i flux measurement for GEMS_N3783_6 is $\sim 45$ per cent lower than the HICAT flux measurement (HIPASSJ1149-38a). In this case, the HICAT spectrum has an extremely uneven baseline and thus the GEMS flux is more reliable. In the case of GEMS_N3783_9, the GEMS flux is 30\% higher than the HICAT flux (HIPASSJ1135-38). In this case, both GEMS and HICAT H I spectrum have a good baseline so it is unclear what causes the discrepancy in the flux. An independent measurement will be needed to confirm the flux for this source. As our rms noise is lower, we adopt the GEMS derived flux for this source.

\subsection{X-ray characteristics of the group}

X-ray observations of the NGC 3783 group were obtained from the ROSAT PSPC archive, and the data reduction is described in OP04. X-ray images for each galaxy group in the GEMS survey are available in Forbes et al. (2006). The resolution of the $\mathrm{X}$-ray images are $30^{\prime \prime}$. The $\mathrm{X}$-ray emission is centred on the galaxy NGC 3783, and OP04 find NGC 3783 itself to have an extended X-ray halo, consistent with intragroup X-ray emission (see Figure 3). However, they were unable to fit a 2-component model to the X-ray distribution, and thus were unable to distinguish the galaxy emission from the group $\mathrm{X}$-ray emission. The extent of the X-ray emission in NGC 3783 is $69 \mathrm{kpc}$, which is low compared to other loose groups that typically have group X-ray halos greater than $100 \mathrm{kpc}$ in size (Mulchaey \& Zabludoff 1998). The X-ray luminosity for NGC 3783 is low at $\log L_{X}=40.76 \pm 0.11$ $\operatorname{ergs~} \mathrm{s}^{-1}$.

\subsection{Dynamical and optical properties of the NGC 3783 group}

Using the NED and 6dFGS DR2 databases, we find 44 galaxies within the region of our $\mathrm{H}$ I datacube, and between velocities $2500-3500 \mathrm{~km} \mathrm{~s}^{-1}$. Of these, $31(\sim 70 \%)$ have their only redshift from the 6dFGS DR2. We use all previously catalogued galaxies in the region, along with the new HI detections, to investigate the characteristics of this group. The velocities used in the calculations are 6dFGS DR2 velocity where available $(79 \%)$, then velocities from NED where available $(15 \%)$ and finally $\mathrm{H}$ i velocities (6\%). Parameters for the NGC 3783 group are shown in Table 4

To determine the centre of the NGC 3783 group, we use a luminosity-weighted mean, based on the $K$-band magnitudes of the galaxies where available from 2MASS. Those galaxies without a known $K$-band magnitude are given a magnitude of 13.5 (as in Brough et al. 2006). All galaxies within the extent of the $\mathrm{H}$ I cube, and with velocities $2500<v_{\text {sys }}<3500 \mathrm{kms}^{-1}$ are included in the calculation. The luminosity weighted centre is $\alpha, \delta(\mathrm{J} 2000)=$ 

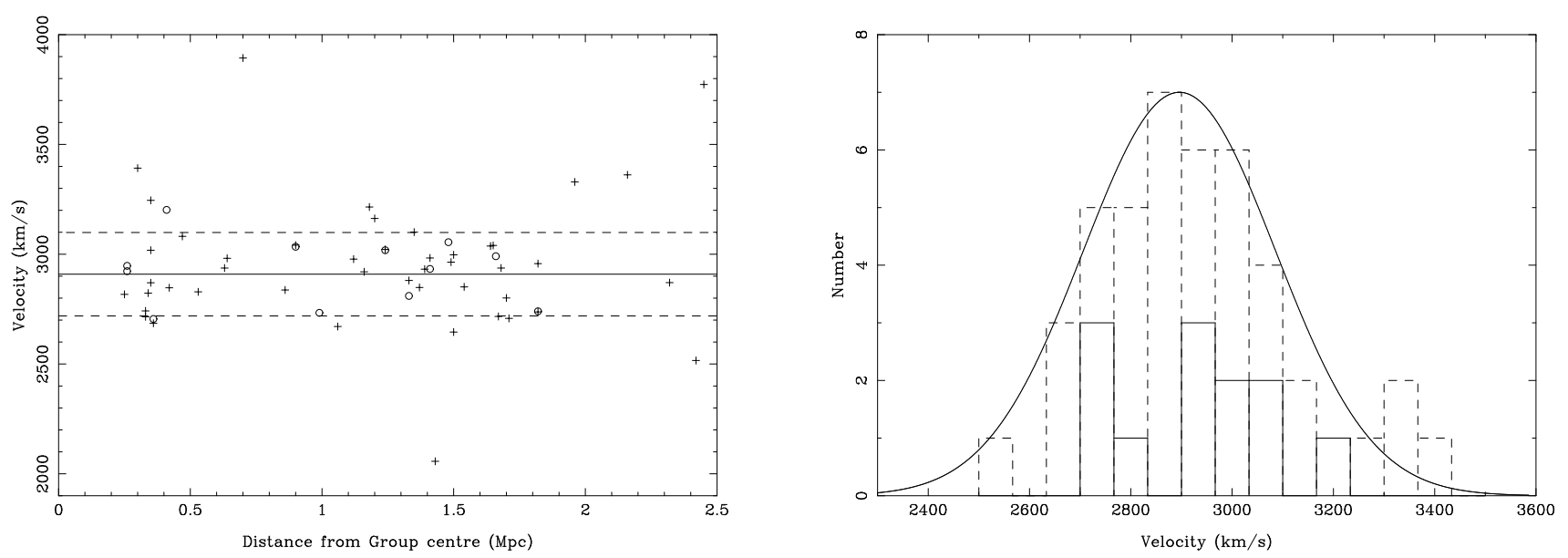

Figure 4. Left: Velocity-distance plot for the NGC 3783 group, using the luminosity-weighted centre. The crosses mark the NED and 6dFGS DR2 sources, and the open circles are the H I detections. The solid line is the mean velocity of the group, $2903 \mathrm{~km} \mathrm{~s}^{-1}$, and the dashed lines are the velocity dispersion, $190 \mathrm{~km} \mathrm{~s}^{-1}$. Right: Velocity histogram of the NGC 3783 group, with Gaussian of width 190 $\mathrm{km} \mathrm{s}^{-1}$, and centre $2903 \mathrm{~km} \mathrm{~s}^{-1}$ overlaid. The dashed line shows the velocity distribution for all galaxies in the region, and the solid histogram shows the $\mathrm{H}$ I detected sources.

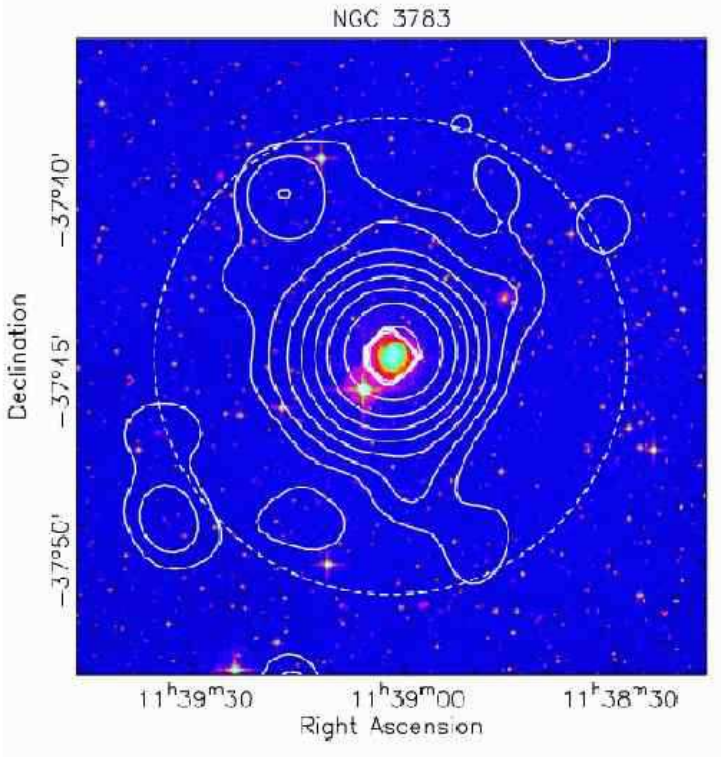

Figure 3. X-ray contours overlaid on the optical image of NGC 3783. The dashed circle indicates the detectable extent of the group X-ray emission (Forbes et al. 2006).

11:37:12,-37:30:57.6, and is marked on Figure प This centre is closest to the galaxy NGC 3783 itself, at a distance of $267 \mathrm{kpc}$ away. As NGC 3783 is the largest galaxy in the group, and with an extended X-ray halo it is expected to lie closest to the group dynamical centre.

Figure 4 shows the velocity-distance distribution for the NGC 3783 group, with the mean velocity and velocity dispersion for the group overlaid. The mean velocity and velocity dispersion were calculated following Beers et al. (1990). We calculate a mean velocity of $2903 \pm 26 \mathrm{~km} \mathrm{~s}^{-1}$ and dispersion of $190 \pm 24 \mathrm{~km} \mathrm{~s}^{-1}$ for the group. The velocity of the galaxy NGC 3783 is very close to the mean velocity of the group, at $2916 \mathrm{~km} \mathrm{~s}^{-1}$ (from the $\mathrm{H}$ I data). Comparing these values to those in the literature, in the original determination of the group, Giuricin et al. (2000) found a median group velocity of $2854 \mathrm{~km} \mathrm{~s}^{-1}$, which is slightly lower than our value. OP04 search for group members for NGC 3783 using NED galaxies within a radius of $0.25 \mathrm{Mpc}$ (the radius at which the density of the group fall to 500 times the critical density of the Universe, calculated from the X-ray temperature of the group), and $\pm 3 \sigma$ of the velocity dispersion of the group. However, under these strict criteria, they find no other catalogued galaxies lie in the group region. They quote the group velocity as $2917 \mathrm{~km} \mathrm{~s}^{-1}$, which is the optical velocity of the galaxy NGC 3783 itself. Brough et al. (2006) use a friends-of-friends algorithm to determine the members and characteristics of the NGC 3783 group, and find the group has nine members, a mean group velocity of $2826 \pm 14 \mathrm{~km} \mathrm{~s}^{-1}$, and velocity dispersion of $118 \pm 37 \mathrm{~km} \mathrm{~s}^{-1}$.

The velocity distribution of the galaxies is also shown in Figure 4 with a Gaussian centred on $2903 \mathrm{~km} \mathrm{~s}^{-1}$, with a width of $190 \mathrm{~km} \mathrm{~s}^{-1}$ overlaid. The distribution of the galaxies appears normal, with a slight skewness to higher velocities. The velocity distribution of $\mathrm{H}$ I detected galaxies appears the same as for all galaxies in the region.

\section{HIGH RESOLUTION OBSERVATIONS OF NGC 3783, AND A NEW DWARF GALAXY}

We obtained ATCA archive H I observations of the galaxy NGC 3783 (see Table 2 for details). Along with the detection of NGC 3783, we detected GEMS_N3783_8, at a distance of $\sim 15^{\prime}(\sim 160 \mathrm{kpc})$ from NGC 3783, and at a velocity of $2983 \mathrm{~km} \mathrm{~s}^{-1}$. The position of the H I emission from the ATCA data is $\alpha, \delta(\mathrm{J} 2000)=11: 38: 01.8,-37: 57: 59$, which is directly centred on a previously uncatalogued dwarf galaxy. An ATCA H I map showing NGC 3783 and the new dwarf 
Table 5. New group members discovered in the NGC 3783 Group. The positions and velocities were derived from ATCA follow-up data, apart from GEMS_N3783_10, where these values were derived from the Parkes data. The columns are as follows: (1) GEMS source name, (2) Previous optical ID, (3) R.A., Dec (J2000), (4) Systemic Velocity, (5) H i mass derived from the Parkes data, (6) H I mass derived from ATCA data.

\begin{tabular}{|c|c|c|c|c|c|}
\hline $\begin{array}{l}\text { GEMS Name } \\
\text { (1) }\end{array}$ & $\begin{array}{l}\text { Optical ID } \\
\qquad(2)\end{array}$ & 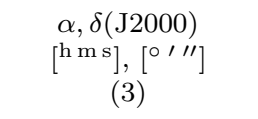 & $\begin{array}{l}\text { Velocity } \\
\mathrm{km} \mathrm{s}^{-1} \\
\quad(4)\end{array}$ & $\begin{array}{c}\text { H I Mass }(\mathrm{PKS}) \\
10^{8} M_{\odot} \\
(5)\end{array}$ & $\begin{array}{c}\text { H i Mass (ATCA) } \\
10^{8} M_{\odot} \\
(6)\end{array}$ \\
\hline GEMS_N3783_2 & $\cdots$ & $11: 31: 27,-36: 18: 37$ & $2731 \pm 4$ & $3.8 \pm 1.3$ & $1.9 \pm 0.3$ \\
\hline GEMS_N3783_8 & $\cdots$ & $11: 38: 02,-37: 57: 59$ & $2983 \pm 8$ & $21 \pm 2.1$ & $7.0 \pm 2.0$ \\
\hline GEMS_N3783_10 & ESO 319 -G 020 & $11: 26: 06,-37: 51: 26$ & $2810 \pm 5$ & $7.2 \pm 1.5$ & $\ldots$ \\
\hline ATCA_1134-37 & & $11: 34: 02,-37: 14: 15$ & $3141 \pm 5$ & $\ldots$ & $2.4 \pm 0.6$ \\
\hline
\end{tabular}

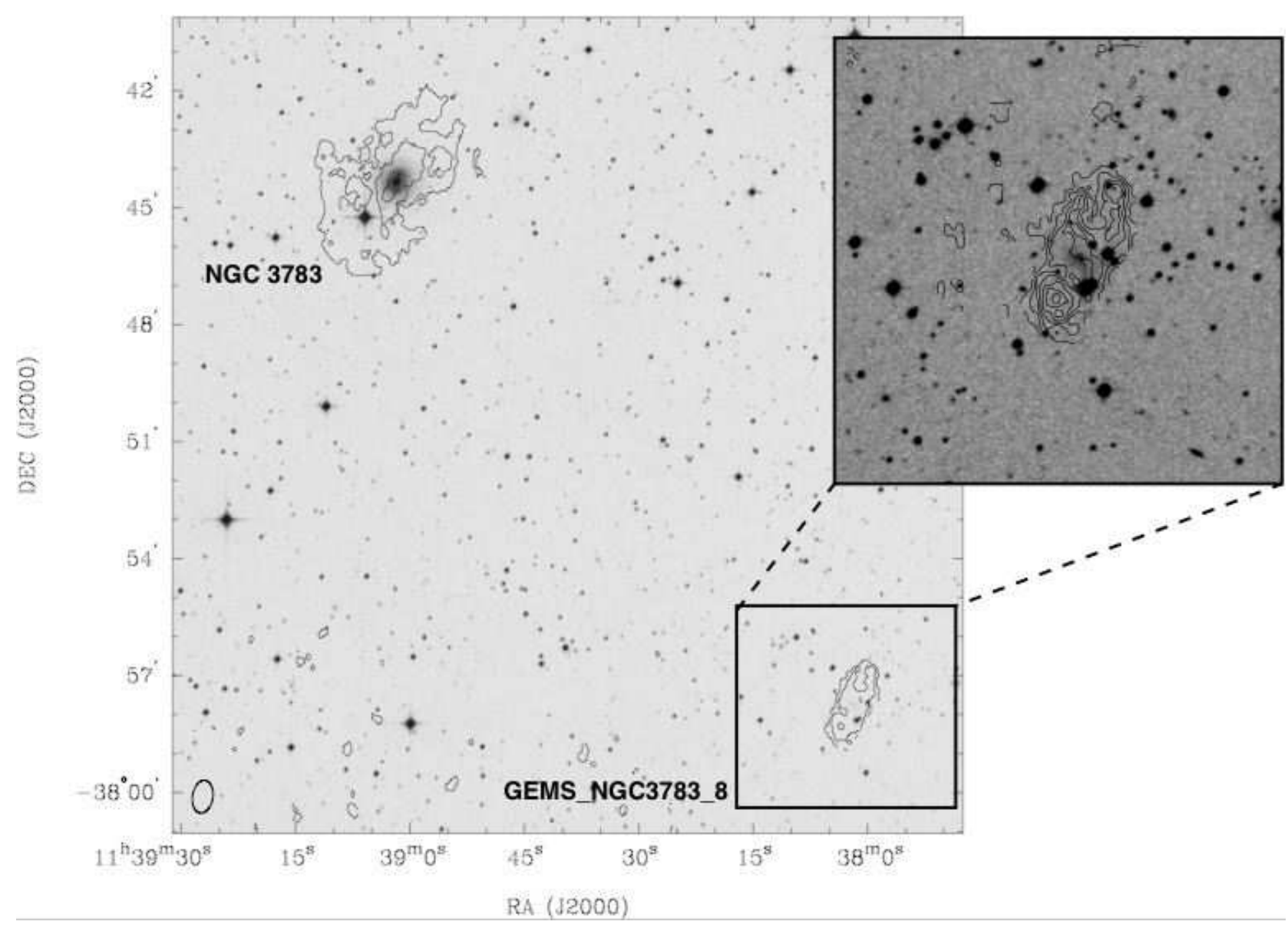

Figure 5. Velocity integrated H I flux distribution for NGC 3783 (GEMS_N3783_7; top left) and GEMS_N3783_8 (bottom right), overlaid on the DSS II $B$-band image. The contour levels in the main image are $0.15,0.3,0.6 \mathrm{Jy} \mathrm{km} \mathrm{s}{ }^{-1}$. The insert is a blow-up of GEMS_N3783_8, overlaid on the DSS II $B$-band image, and the contour levels are $0.15,0.2,0.3,0.4,0.5,0.6 \mathrm{Jy} \mathrm{km} \mathrm{s}^{-1}$. The ATCA beam is shown in the bottom left of the main image.

galaxy is shown in Figure 5 Other faint galaxies are visible in the optical image of the observed field, but not detected in H I. GEMS_N3783_8 lies near the edge of the ATCA primary beam. For clarity, the noisy edge of the data was masked out in this image. The H I mass for the new dwarf galaxy measured in the ATCA data is $7 \pm 2 \times 10^{8} M_{\odot}$, compared to an H I mass of $2.1 \pm 0.25 \times 10^{9} M_{\odot}$ from our Parkes data, thus it appears that this dwarf galaxy has an extended H I component that was resolved out in the ATCA observations. It should be noted that as GEMS_N3783_8 lies near the edge of the primary beam, the ATCA flux may be underestimated.
The ATCA position of GEMS_N3783_8 is indicated in Figure 1 and the parameters for GEMS_N3783_8 are given in Table 5] GEMS_N3783_8 lies between NGC 3783 and ESO $320-\mathrm{G} 013$ both spatially (190kpc and $120 \mathrm{kpc}$ projected separation respectively), and in velocity (166 and $35 \mathrm{~km} \mathrm{~s}^{-1}$ respectively). 


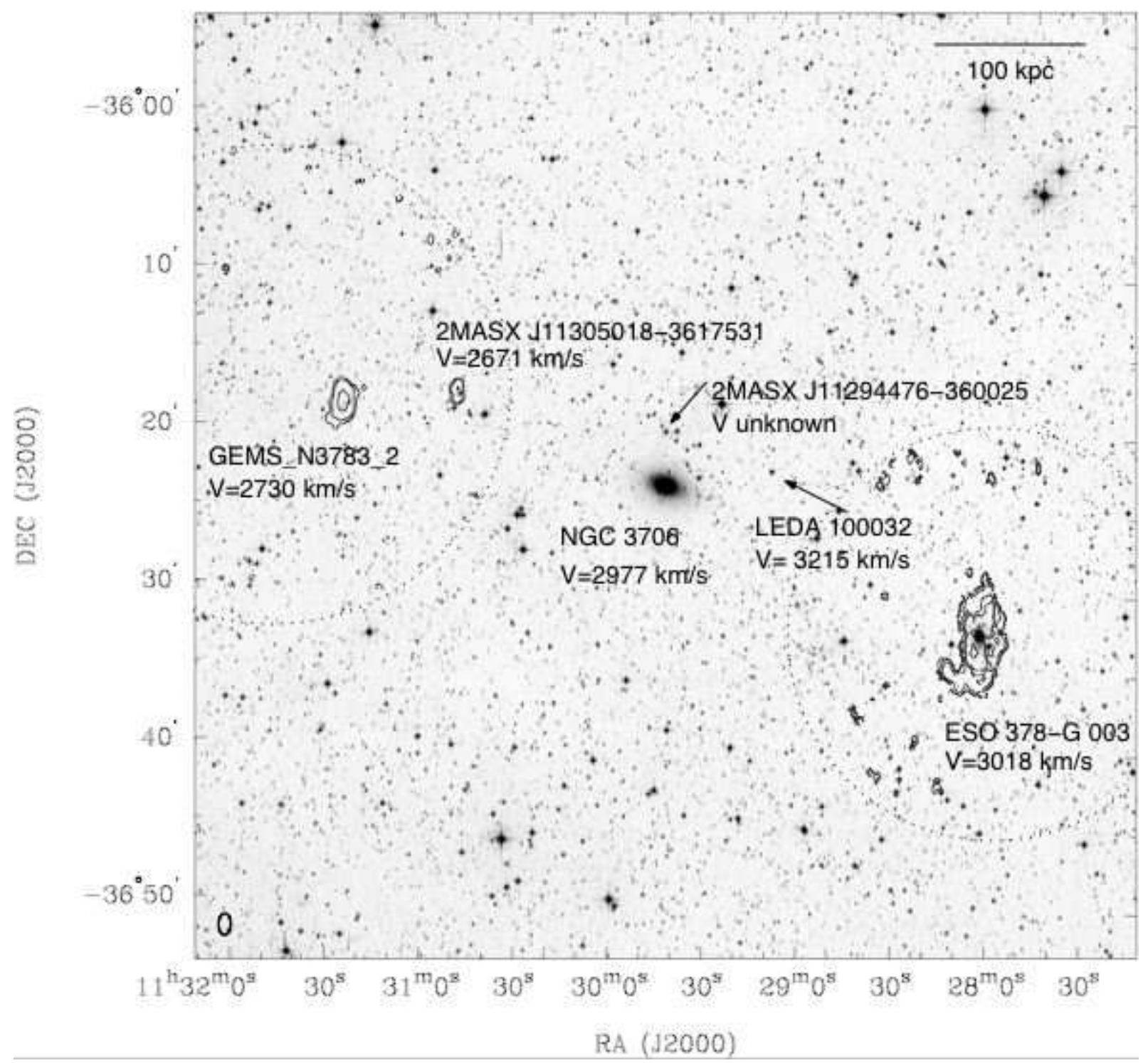

Figure 6. Neutral hydrogen distribution of GEMS_N3783_2 and ESO 378-G 003 (GEMS_N3783_3), overlaid on the DSS II R-band image. The contours show the H I emission, and the levels are $0.15,0.2,0.4,0.8,1 \mathrm{Jy} \mathrm{km} \mathrm{s}^{-1}$. The ATCA beam of $88^{\prime \prime} \times 50^{\prime \prime}$ is shown in the bottom left corner. Two separate ATCA pointings were used, and the noisy edge of the primary beam corrected H I datacubes have been masked out indicated by the dashed lines in the image. Along with GEMS_N3783_2, we detect 2MASX J11305018-3617531 in H I. Heliocentric velocities are indicated.

Table 4. Characteristics of the NGC 3783 group

\section{Distance $[\mathrm{Mpc}]$}

Luminosity Weighted Centre $[\alpha, \delta(\mathrm{J} 2000)] \quad$ 11:37:12,-37:30:57.6

Total number of galaxies

Number of H i detections

Mean velocity, $v\left[\mathrm{~km} \mathrm{~s}^{-1}\right]$

Velocity dispersion, $\sigma_{v}\left[\mathrm{~km} \mathrm{~s}^{-1}\right]$

$\log L_{X}\left[\mathrm{ergs} \mathrm{s}^{-1}\right]$

Total H I mass $\left[M_{\odot}\right]$

${ }^{\dagger}$ from Osmond \& Ponman (2004)

\section{INTRA-GROUP H I GAS}

There is one other HI detection that does not correspond with any previous NED or 6dFGS DR2 optically catalogued galaxy, GEMS_N3783_2. However, unlike GEMS_N3783_8, inspection of optical images obtained from the Second Generation Digital Sky Survey (DSS II), at the position of the $\mathrm{H}$ I detection showed no obvious corresponding optical emission.

We obtained higher resolution follow-up observations from the ATCA for GEMS_N3783_2, described in Table 2 The ATCA velocity integrated H I flux density map overlaid on the corresponding DSS II $R$-band image is shown in Figure 6 and a close-up of the region is shown in Figure 7 The peak column density of GEMS_N3783_2 is $7.5 \times 10^{19} \mathrm{~cm}^{-2}$. GEMS_N3783_2 has a total H I mass of $M_{\mathrm{HI}}=3.8 \pm 1.3 \times 10^{8}$ $M_{\odot}$, determined from the Parkes observations. The ATCA H I map is unresolved with the ATCA beam size of $88^{\prime \prime} \times 50^{\prime \prime}$. The H I parameters for GEMS_N3783_2 are given in Table 6 The H I mass detected by the ATCA is $1.9 \pm 0.3 \times 10^{8} M_{\odot}$, 


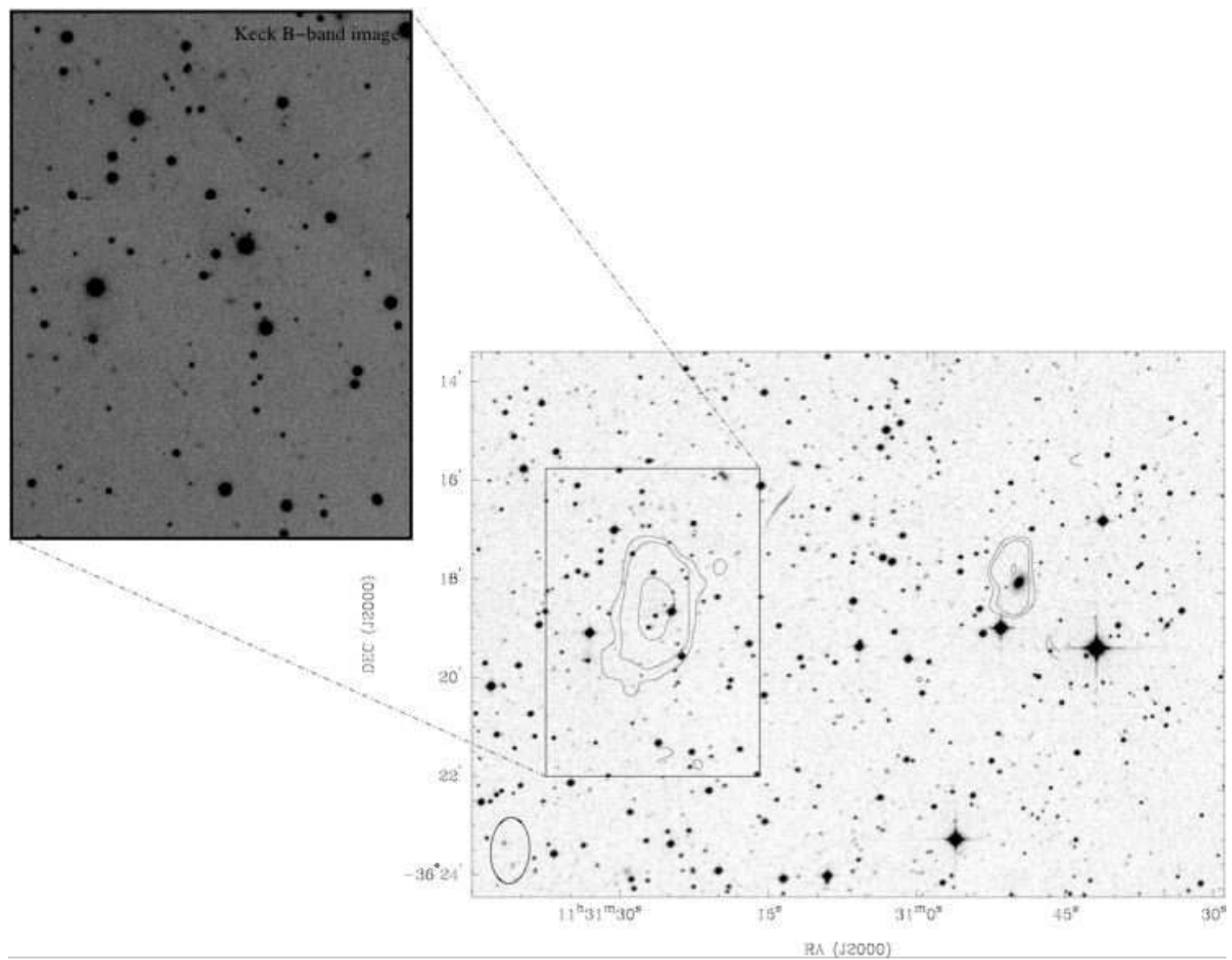

Figure 7. Neutral hydrogen distribution of GEMS_N3783_2 (left) and 2MASX JJ11305018-3617531 (right), overlaid on the DSS II $R$-band image. The contour levels are $0.15,0.2,0.3 \mathrm{Jy} \mathrm{km} \mathrm{s}^{-1}$. The ATCA beam of $88^{\prime \prime} \times 50^{\prime \prime}$ is shown in the bottom left corner. The insert shows the $B$-band Keck image for the region surrounding GEMS_N3783_2.

slightly lower than the Parkes observations, indicating we are missing some extended H i emission. We made optical observations in the region of this object with the Keck telescope on 2005 Feb. 9 (see Figure (7). Images in $R$ and $B$ bands do not show the presence of any low surface brightness galaxy down to a limiting surface brightness of $B \sim 22$ mag $\operatorname{arcsec}^{-2}$.

There are several small, faint optical sources within the HI emission region for GEMS_N3783_2. We obtained a short service time observation with the $2 \mathrm{dF}$ multi-fibre spectrograph on 2005 May 4 to obtain redshifts for nearby bright, resolved optical sources. The $2 \mathrm{dF}$ spectrograph covers a 2 degree field which could encompass the region about GEMS_N3783_2 in the one pointing. Most of the sources we observed did not contain emission or absorption lines for us to determine a redshift from. No sources were found at the group velocity.

What is the origin of GEMS_N3783_2? We have little information as yet on the structure or velocity field of this H I region, making it hard to determine the origin by studying it. Examining Figure 6] The nearest bright galaxy to GEM_N3783_2 is the early-type galaxy NGC 3706. There are several nearby dwarf galaxies, and the nearest gas-rich spiral galaxies are $>500 \mathrm{kpc}$ away. We have obtained high resolution ATCA H i observations of the nearest spiral galaxies.
The H I distribution of ESO 378-G 003 is shown in Figure 6 and Figure 8 and the H I distribution of ESO 378-G 011 is shown in Figure 9

ESO 378-G 003 lies at a projected distance of $\sim 235$ kpc from NGC 3706, and 450 kpc from GEMS_N3783_2. ESO 378-G 003 shows an extended $\mathrm{H}$ I distribution, which is distorted on the SE side of the galaxy. The velocity contours shown in Figure 8 show typical spiral rotation in the undisturbed side of the galaxy, while the extended part shows irregular rotation, indicating a tidal interaction.

Figure 9 shows the $\mathrm{HI}$ distribution for ESO 378-G 011 overlaid on the DSS II $R$-band image. In our ATCA HI datacube, we also detect a previously uncatalogued dwarf galaxy, ATCA_1134-37, at the position $\alpha, \delta(\mathrm{J} 2000)$ $=11: 34: 03,-37: 14: 22$ ). ESO 378-G 011 lies at a projected distance of $\sim 570 \mathrm{kpc}$ from GEMS_N3783_2, and the H I distribution for the galaxy appears regular.

\section{DISCUSSION}

The NGC 3783 group is a loose group of galaxies, with diffuse X-ray emission centred on the galaxy NGC 3783 itself. What is the evolutionary state of this group - for example, is it virialised? The extent of the X-ray emission is 69 $\mathrm{kpc}$, which is low for intra-group X-ray emission, and only a 


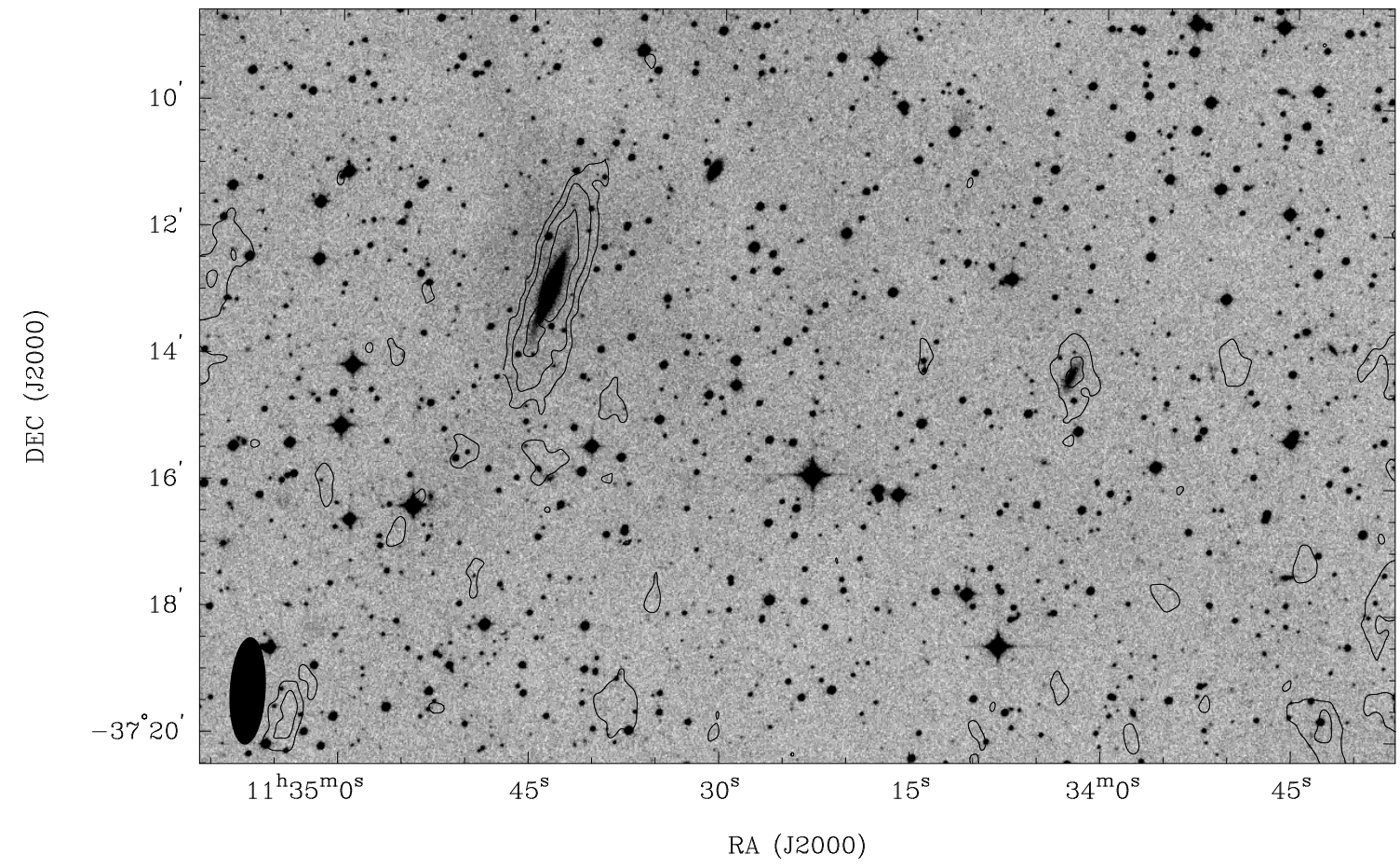

Figure 9. Neutral hydrogen distribution of ESO 378-G 011 (left), and the small, previously uncatalogued dwarf galaxy ATCA_1134-37 (right), overlaid on the DSS II $R$-band image. The contour levels are $0.5,1,2,3 \mathrm{Jy} \mathrm{km} \mathrm{s}^{-1}$.

Table 6. Neutral hydrogen parameters of GEMS_N3783_2.

\begin{tabular}{lcc}
\hline & Parkes & ATCA \\
\hline Central Position $[\alpha, \delta(\mathrm{J} 2000)]$ & $11: 31: 32,-36: 18: 53$ & $11: 31: 27,-36: 18: 37$ \\
H I Mass $\left[10^{8} M_{\odot}\right]$ & $3.8 \pm 1.3$ & $1.9 \pm 0.3$ \\
Velocity $\left[\mathrm{km} \mathrm{s}^{-1}\right]$ & 2730 & 2731 \\
$\Delta v_{20}\left[\mathrm{~km} \mathrm{~s}^{-1}\right]$ & 116 & 50 \\
$\Delta v_{50}\left[\mathrm{~km} \mathrm{~s}^{-1}\right]$ & 106 & 40 \\
\hline
\end{tabular}

single component fit to the surface brightness profile of the X-ray emission was possible. For comparison, Mulchaey \& Zabludoff (1998) find that for nine X-ray detected groups it was possible to make a two component fit to the surface brightness of the X-ray emission, with the first component extending 20-40 kpc, and corresponding to emission from the galaxy, and the second component extending 100-300 $\mathrm{kpc}$, and corresponding to diffuse intra-group X-ray emission. The extent of the X-ray emission in the NGC 3783 group could be interpreted in two ways: The X-ray emission could emanate solely from the Seyfert galaxy NGC 3783 itself, or the X-ray emission could be from the combination of emission from NGC 3783, and the group potential of a newly forming galaxy group, perhaps one that is not yet virialised. Looking at the velocity distribution of the group, it is nearly normally distributed, usually a sign of a relaxed system of galaxies. However, the velocity-distance plot shown in Figure 4 does not look like a typical virialised group. The galaxy NGC 3783 itself is the closest galaxy to the luminosity weighted centre of the group, although it is 267 $\mathrm{kpc}$ from this centre, which is unusual for a virialised group (Brough et al. 2006). NGC 3783 lies at the mean velocity of the group. This group seems to display some characteristics of a virialised group, however the fact that the X-ray emission is centred on a late-type galaxy, and is offset from the centre of the group indicates that this may be an example of a group in the early stages of evolution.

In our neutral hydrogen survey of the NGC 3783 group, we found several new group members, and one region of $\mathrm{HI}$ emission that appears to have no stars associated with it. While there are many examples of isolated neutral hydrogen that has been removed from galaxies (e.g. HI rogues gallery (Hibbard et al. 2001a): http://www.nrao.edu/astrores/HIrogues/), this is one of the most extreme examples of isolated $\mathrm{H}$ I ever found. To date, all neutral hydrogen that has been found in emission can be associated with either galaxies, regions of stars or star formation.

There are three possible explanations for the existence of GEMS_N3783_2 in the NGC 3783 galaxy group. Either this is an extremely low surface brightness galaxy that we have been unable to detect optically yet, GEMS_N3783_2 is comprised of gas that has been removed from a galaxy in 


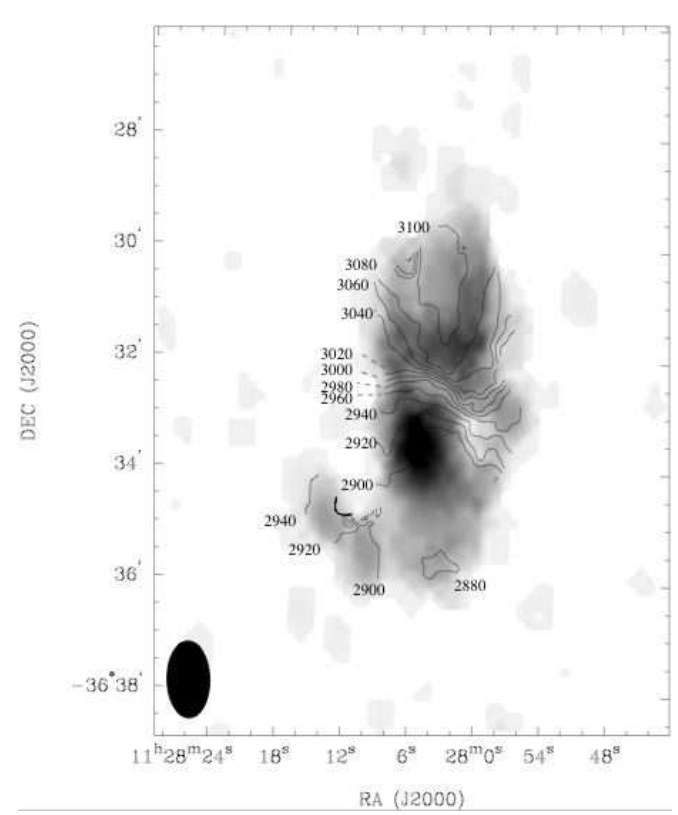

Figure 8. Neutral hydrogen distribution of the spiral galaxy ESO 378-G 003 (greyscale), with velocity contours overlaid. ESO 378G 003 shows signs of tidal interaction in the SE part of the galaxy, with an irregular velocity field, and an extension in the $\mathrm{H}$ I emission.

the past, or GEMS_N3783_2 is a primordial cloud of neutral hydrogen which has not formed stars.

If GEMS_N3783_2 is a low surface brightness galaxy, then the limits placed by our Keck observations are such that the central surface brightness must be less then 22 mag $\operatorname{arcsec}^{-2}$ in the $B$-band. Assuming a source size of about $10^{\prime \prime}$ (e.g. the size of the nearest dwarf galaxy to GEMS_N3783_2, 2MASX J11305018-3617531), then we would have detected a typical dwarf galaxy in our optical imaging. However, we are not sensitive to an extended low surface brightness galaxy, and deeper optical imaging is needed to confirm there is no stellar component to GEMS_N3783_2.

GEMS_N3783_2 may be a remnant of stripping of a gasrich galaxy. It is unlikely that GEMS_N3783_2 was formed through ram pressure stripping, as the hot gas in the NGC 3783 group is confined to the region around NGC 3783 itself, and that the projected distance of GEMS_N3783_2 from the X-ray emission is $>500 \mathrm{kpc}$. GEMS_N3783_2 may have formed through a tidal interaction between a gas-rich galaxy, and one or more other galaxies in the group.

The nearest gas-rich galaxy to GEMS_N3783_2 is 2MASX J11305018-3617531, for which we detected an $\mathrm{HI}$ mass of $\sim 10^{7} M_{\odot}$ in our ATCA observations. Given the H I mass of GEMS_N3783_2 at $\sim 4 \times 10^{8} M_{\odot}$ is typical of a dwarf irregular galaxy (eg: Salzer et al. 2002; Stil \& Israel 2002; Hoffman et al. 1996), perhaps 2MASX J11305018-3617531 has been tidally influenced by NGC 3706, removing the majority of the $\mathrm{H}$ I from the galaxy. This seems unlikely as we do not detect a bridge of H I joining GEMS_N3783_2 and 2MASX J11305018-3617531, and to remove the majority of $\mathrm{H}$ I from a dwarf galaxy while leaving the stars intact would require an extremely extended $\mathrm{H}_{\mathrm{I}}$ distribution of the dwarf galaxy.

The nearest gas-rich spirals to GEMS_N3783_2 are ESO
378-G 003 and ESO 378-G 011, at a projected separation of $\sim 450$ and $\sim 570 \mathrm{kpc}$ respectively. Given the large projected separation, and apparently undisturbed $\mathrm{H}$ I distribution of ESO 378-G 011, we do not consider that it is the origin of GEMS_N3783_2. On the other hand, ESO 378-G 003 is closer to GEMS_N3783_2, and importantly, is also very close to the bright early-type galaxy NGC 3706. ESO 378-G 003 displays evidence of tidal interaction in its irregular $\mathrm{H}$ I and velocity distribution on one side of the galaxy. If GEMS_N3783_2 was formed from the interaction of NGC 3706 and ESO 378-G 003, then using the difference in velocity of GEMS_N3783_2 and ESO 378-G 003, and the projected separation of 450 $\mathrm{kpc}$, the time-scale for the interaction is $\sim 1.5$ Gyr. Of the many possibilities, this seems the most likely, but it requires deeper observations to confirm. Deeper observations might uncover further $\mathrm{H}$ I in the system, as in the case of the Leo cloud (Schneider 1985), and the NGC 1490 system (Oosterloo et al. 2004).

Finally, could GEMS_N3783_2 be an isolated primordial H I cloud, that has lived quiescently forming no stars? Cosmological simulations of groups have predicted many more dark matter halos than optical galaxies are seen observationally (eg: D'Onghia \& Lake 2004; Moore et al. 1999; Klypin et al. 1999). Perhaps GEMS_N3783_2 is an example of such a dark halo, containing primordial H I gas? While not impossible, this option does seem unlikely. Of our H I survey of 16 galaxy groups, GEMS_N3783_2 is the only detection that we have found which does not have an obvious galaxy associated with it. If GEMS_N3783_2 is primordial material, then it is the only example we have found of such an object in our H I survey, and thus the number of these objects appears to be very low. We note, that our survey of 16 groups has a mass limit of $\gtrsim 5 \times 10^{8} M_{\odot}$, so we are unable to say anything about the population of lower mass $\mathrm{H}$ I clouds, however other authors have not found any low mass H I clouds in deep H I studies of loose groups (Pisano 2004; Zwaan 2001).

\section{CONCLUSIONS}

We have made a wide-field H I survey of the NGC 3783 galaxy group, using the Parkes radiotelescope. We found $12 \mathrm{HI}$ detections in the region, of which one is a region of extended $\mathrm{H}$ I emission that we cannot match with corresponding optical emission. We believe the origin of this $\mathrm{H}$ I region is likely to be tidal debris rather than ram-pressure stripped, or primordial H I. We found two previously uncatalogued dwarf galaxies - one from our Parkes observations (GEMS_N3783_8), and one from high resolution ATCA observations (ATCA_1134-37). We used the 6dFGS DR2 and NED databases to find previously catalogued galaxies in the region, and determine parameters for the NGC 3783 group. We calculate a mean velocity for the group of $2903 \pm 26$ $\mathrm{kms}^{-1}$, and velocity dispersion of $190 \pm 24 \mathrm{kms}^{-1}$. The NGC 3783 group is a rare example of a group at the early stage of its evolution. Comparison with other GEMS groups will help in determining the evolutionary path of galaxies within groups in general. 


\section{ACKNOWLEDGMENTS}

We thank David Barnes for invaluable help in reducing the Parkes narrow-band data, and for making modifications to the reduction software. We thank Rob Sharp for taking the 2dF observations of GEMS_N3783_2 optical candidates. We acknowledge Heath Jones and Matthew Colless for providing helpful information on the 6dFGS data. We thank Jean Brodie for the Keck observations of GEMS_N3783_2. John Osmond and Trevor Ponman are acknowledged for helping us with the X-ray data. We thank John Reynolds for support with the Parkes observations. We thank the anonymous referee for useful comments.

This research has made use of the NASA/IPAC Extragalactic Database (NED) which is operated by the Jet Propulsion Laboratory, California Institute of Technology, under contract with the National Aeronautics and Space Administration. This publication makes use of the Two Micron All Sky Survey (2MASS) which is a joint project of the University of Massachusetts and the Infrared Processing and Analysis Centre/California Institute of Technology, funded by the National Aeronautics and Space Administration and the National Science Foundation. The Digitized Sky Survey was produced at the Space Telescope Science Institute under U.S. Government grant NAG W-2166.

\section{REFERENCES}

Abadi M. G., Moore B., Bower R. G. 1999, MNRAS, 308, 947

Banks G. D. et al. 1999, ApJ, 524, 612

Barnes J. E., Hernquist L. 1996, ApJ, 471, 115

Beers T. C., Flynn K., Gebhardt K. 1990, AJ, 100, 32

Bekki K., Koribalski B. S., Kilborn V. A. 2005a, MNRAS, 363,21

Bekki K., Koribalski B. S., Ryder S. D., Couch W. J. 2005b, 357, 21

Blank D. L., Cram, L. E. 2000, MNRAS, 312, 247

Brough, S. et al. 2006, MNRAS, in press, astro-ph/0605279 Davies J. et al. 2004, MNRAS, 349, 922

Djorgovski S. 1990, AJ, 99, 31

D’Onghia E., Lake G. 2004, ApJ, 612, 628

Forbes D. A. et al. 2006, PASA, 23, 38

Garcia-Barreto J. A., Combes F., Koribalski B., Franco J. 1999, A\&A, 348, 685

Giovanelli R. \& Haynes M. P. 1989, ApJ, 346, 5

Giuricin G., Marioni C., Ceriani L., Pisani A. 2000, ApJ, 543,178

Haynes M. P. 1981, AJ, 86, 112

Haynes M. P. \& Giovanelli R. 1984, AJ, 89, 758

Hibbard J. E. \& Yun M. S. 1999, ApJ, 522, 93

Hibbard J. E., van Gorkom J. H., Rupen M. P., Schiminovich D. 2001a, ASP Conference Series Vol 240, "Gas and Galaxy Evolution", eds J. E. Hibbard, J.H. van Gorkom, M.P. Rupen, 657

Hibbard J. E., van der Hulst, J. M., Barnes, J. E., Rich, R. M. 2001b, AJ, 122, 2969

Hoffman G. L., Salpeter E. E., Farhat B., Roos T., Williams H., Helou G. 1996, ApJ, 105, 269

Jones D. H. et al. 2005, PASA, 22, 277

Kilborn V. A., Koribalski B. S., Forbes D. A., Barnes D.

G., Musgrave R. C. 2005, MNRAS, 356, 77
Kilborn V. A., et al. 2006, in prep.

Klypin A., Kravstov A. V., Valenzuela O., Prada F. 1999, ApJ, 522, 82

Koribalski B. \& Manthey E. 2005, MNRAS, 358, 202

Koribalski B. \& Dickey, J. M. 2004, MNRAS, 348, 1255

Koribalski B. et al. 2004, AJ, 128, 16

Li J. G., Seaquist E. R. 1994, AJ, 107, 1953

Mathews L. D., Gallagher J. S., Littleton J. E. 1995, AJ, 110,581

Meyer M. J. et al. 2004, MNRAS, 350, 1195

Minchin R. F. et al. 2005, ApJ, 622, 21

Moore B., Ghigna S., Governato F., Lake G., Quinn T., Stadel J., Tozzi P. 1999, ApJ, 524, 19

Mulchaey J. S., Zabludoff A. I. 1998, ApJ, 496, 73

Omar A., \& Dwarakanath K. S. 2005, JApA, 26, 1

Oosterloo T. \& van Gorkom J. 2005, A\&A, 437, 190

Oosterloo T., Morganti R., Sadler E. M., Ferguson A., van der Hulst T., Jerjen H. 2004, IAU Symposium series, Vol. 217, editors P.-A. Duc, J. Braine, E. Brinks, 486

Osmond J. P. F., Ponman T. J. 2004, MNRAS, 350, 1511

Pisano D. J., Barnes D. G., Gibson B. K., StaveleySmith L., Freeman K. C., Kilborn V. A. 2004, ApJL, 601, 17

Ryder S. D. et al. 2001, ApJ, 555, 232

Salzer J. J., Rosenberg J. L., Weisstein E. W., Mazzarella J. M., Bothun G. D. 2002, ApJ, 124, 191

Schneider S. 1985, ApJL, 288, 33

Stil J. M. \& Israel F. P. 2002, A\&A, 389, 29

Toomre A. \& Toomre J. 1972, ApJ, 178, 623

Yun M. S., Ho P. T., Lo K. Y. 1994, Nature, 372, 530

Zabludoff A. I. \& Mulchaey J. S. 1998, ApJ, 496, 39

Zwaan M. A. 2001, MNRAS, 325, 1142 